\title{
TRATAMENTO DO LIXIVIADO DE ATERRO DE RESÍDUOS SÓLIDOS URBANOS POR PROCESSO FENTON
}

\author{
Victor Wegner Maus ${ }^{1}$, Adilson Ben da Costa ${ }^{2}$, Afranio Almir Righes ${ }^{3}$ \\ 1 Mestrado em Modelagem Computacional - Universidade Federal de Juiz de Fora, Juiz de Fora, Minas Gerais, Brasil. \\ 2 Departamento de Biologia e Farmácia - Universidade de Santa Cruz do Sul, Santa Cruz do Sul, Rio Grande do Sul, Brasil. \\ 3 Departamento de Engenharia Ambiental - Centro Universitário Franciscano e Diretor do Centro Regional Sul de Pesquisas \\ Espaciais - INPE, CRS/INPE, Santa Maria, Rio Grande do Sul, Brasil.
}

*E-mail:vwmaus@bol.com.br

Recebido em 04 de junho de 2009

Aceito em 31 de junho de 2009

\begin{abstract}
RESUMO
O lixiviado gerado em aterros sanitários pela decomposição dos resíduos sólidos com presença de água de infiltração possui grande potencial poluidor, devido à elevada concentração de substâncias tóxicas e a sua baixa biodegradabilidade. A coagulação química pode ser uma alternativa para o tratamento de efluentes com essas características. O trabalho teve como objetivo avaliar a eficiência do processo Fenton no tratamento de lixiviado gerado em aterros sanitários de resíduos sólidos urbanos. As amostras de lixiviado foram coletadas no aterro controlado do município de Santa Maria - RS. No experimento foram testados cinco tratamentos com diferentes proporções de reagentes $\left(\mathrm{H}_{2} \mathrm{O}_{2}\right.$ e $\left.\mathrm{FeSO}_{4}\right) \mathbf{T H}_{\mathbf{2}} \mathbf{O}_{\mathbf{2}} / \mathbf{F e S O}_{\mathbf{4}}$ : $\mathbf{T}_{\mathbf{0 , 8}, \mathbf{8}}, \mathbf{T}_{\mathbf{2}, \mathbf{0}}, \mathbf{T}_{\mathbf{3 , 0}, \mathbf{0}}, \mathbf{T}_{\mathbf{4 , 0},}$ e $\mathbf{T}_{\mathbf{6}, \mathbf{0}}$. Pelos resultados do experimento com processo Fenton verifica-se uma eficiência média na remoção da demanda química de oxigênio (DQO) de 47,9\%, na turbidez de 53,0\% e na cor de 70,7\%. Os valores médios da demanda bioquímica de oxigênio (DBO) e da condutividade elétrica aumentaram em $31,3 \%$ e $27,1 \%$, respectivamente. No tratamento $\mathbf{T}_{\mathbf{0}, \mathbf{8}}$ constatou-se a maior remoção da DQO e o maior aumento da biodegradabilidade. O processo Fenton aplicado no tratamento de lixiviado reduz a DQO e aumenta a DBO, em outras palavras, aumenta a biodegradabilidade do efluente. Esse processo pode ser utilizado como pré-tratamento de um sistema biológico para efluentes de aterros sanitários.
\end{abstract}

Palavras-chave: percolado. efluente. biodegradabilidade. processo oxidativo avançado. aterro sanitário.

\section{INTRODUÇÃO}

O rápido crescimento populacional aliado ao desenvolvimento tecnológico vem causando freqüentes impactos ambientais. A pressão sobre o meio ambiente tem conseqüências, como: poluição atmosférica, do solo, da água, deslizamentos, enchentes entre outras [1].

Segundo o Instituto Brasileiro de Geografia e Estatística (IBGE) [2], a produção média per capita de resíduos sólidos urbanos para a população brasileira é de 0,74kg.(hab.dia) ${ }^{-1}$. Para o Instituto Brasileiro de Administração Municipal (IBAM) [3], no Brasil admite-se uma geração per capita de resíduos sólidos urbanos variando entre 0,5 e $0,8 \mathrm{~kg}$.(hab.dia) $)^{-1}$. A geração de resíduos sólidos domésticos esta intimamente ligada a capacidade de consumo da população, desta forma a produção per capita de resíduos pode ter sofrido variações nos últimos anos, devido a consecutivos aumentos do consumo das famílias brasileiras [4].

A disposição final de resíduos sólidos urbanos é feita em aterros sanitários, aterros controlados ou vazadouros a céu aberto (lixões). No que diz respeito a implantação de um aterro sanitário deve-se seguir critérios de engenharia e normas operacionais específicas para confinar os resíduos com segurança, visando o controle da poluição ambiental e proteção à saúde pública. Os aterros controlados são locais de despejos de resíduos sólidos, com o cuidado de, após a jornada de trabalho, cobrir esses resíduos com uma camada de terra sem causar danos ou riscos à saúde pública ou à segurança minimizando os impactos ambientais. Por fim, os vazadouros a céu aberto são locais de disposição de resíduos, em bruto, sobre o terreno sem qualquer cuidado ou técnica especial. Essa forma de disposição de resíduos caracteriza-se pela falta de proteção ao meio ambiente e à saúde pública [2].

Do total de resíduos sólidos domésticos gerados no país, $47,1 \%$ é disposto em aterro sanitário, $22,4 \%$ em aterros controlados e $30,5 \%$ em vazadouros a céu aberto, inferindo que $69,5 \%$ dos resíduos gerados são destinados adequadamente em aterros sanitários e/ou controlados. Considerando-se o número total de municípios, os resultados não são tão favoráveis, sendo que $63,6 \%$ utilizam vazadouros a céu aberto como forma de destino final dos resíduos sólidos urbanos, $18,4 \%$ utilizam aterros controlados 
e apenas 13,8\% utilizam aterros sanitários [2]. Segundo Venino [5], o Ministério das Cidades junto ao IBGE estão realizando um novo levantamento sobre a situação do Saneamento Básico no Brasil e estimam sua publicação para final do ano de 2009.

O biogás e o lixiviado, produzidos em aterros sanitários, são aspectos ambientais que, se não gerenciados de forma adequada, podem causar impactos ao meio ambiente. PROSAB [6] apresenta estudos da composição do biogás produzido em um aterro sanitário da Califórnia em percentagem de volume, sendo $44,03 \%$ metano $\left(\mathrm{CH}_{4}\right)$, $34,20 \%$ dióxido de carbono $\left(\mathrm{CO}_{2}\right), 20,81 \%$ nitrogênio $\left(\mathrm{N}_{2}\right)$, $0,52 \%$ oxigênio, o restante, $0,44 \%$, é composto de hidrogênio $\left(\mathrm{H}_{2}\right)$, monóxido de carbono $(\mathrm{CO})$, gás sulfídrico $\left(\mathrm{H}_{2} \mathrm{~S}\right)$, amônia $\left(\mathrm{NH}_{3}\right)$, mercaptanas sulfuradas, propano $\left(\mathrm{C}_{3} \mathrm{H}_{8}\right)$, butano $\left(\mathrm{C}_{4} \mathrm{H}_{10}\right)$, tolueno $\left(\mathrm{C}_{7} \mathrm{H}_{8}\right)$ e benzeno $\left(\mathrm{C}_{6} \mathrm{H}_{6}\right)$. Conforme esse estudo, o gás produzido em maior quantidade no aterro sanitário é o metano que, segundo Baird [7], tem uma contribuição 21 vezes maior para o aquecimento global, quando comparado ao dióxido de carbono.

Segundo Tartari [8], o lixiviado é gerado pela decomposição dos resíduos sólidos na presença de água de infiltração, principalmente da chuva. Essa água fica em contato com os resíduos, solubilizando os produtos da decomposição e por ação natural da gravidade percola pela porosidade existente até encontrar uma camada impermeável, formada por rochas, ou mesmo superfícies previamente preparadas para receber os resíduos, onde acumula e escoa. PROSAB [9] associa impactos nos recursos hídricos causados por substâncias químicas perigosas presentes nos resíduos sólidos. Essas substâncias químicas são carreadas pela água, infiltrando no solo, de onde podem alcançar aquíf́eros subterrâneos, ou ainda corpos d'água, por ação do escoamento superficial. Segundo Sisinno et al. [10], a poluição das águas pelo lixiviado de aterros sanitários pode provocar endemias ou intoxicações, se houver a presença de organismos patogênicos e substâncias tóxicas em níveis acima do permissível.

O lixiviado gerado a partir dos resíduos sólidos tem baixa biodegradabilidade, no entanto grande parte dos aterros sanitários utiliza apenas sistemas biológicos para tratamento desse efluente. Por meio dos dados de demanda química e bioquímica de oxigênio do lixiviado de diferentes aterros sanitários determinados por vários autores, entre eles Lange et al. [11]; Rodrigues [12]; Morais et al. [13] e Santos e Coelho [14], estima-se uma baixa biodegradabilidade para este tipo de efluente. Devido à baixa biodegradabilidade do lixiviado, faz-se necessário o estudo e implantação de sistemas de tratamento capazes de remover tanto a carga orgânica biodegradável (representada pela demanda bioquímica de oxigênio, DBO) como a carga orgânica não biodegradável (representada pela diferença entre a demanda química de oxigênio, DQO e a DBO). O processo oxidativo avançado com uso de reagentes Fenton pode ser uma alternativa para o tratamento desse tipo de efluente. Conforme Nogueira et al. [15], os métodos de oxidação química podem resultar numa quase completa mineralização dos poluentes orgânicos.
A oxidação por processo Fenton utiliza $\mathrm{Fe}^{2+}$ e peróxido de hidrogênio $\left(\mathrm{H}_{2} \mathrm{O}_{2}\right)$, sendo uma tecnologia comprovadamente efetiva para eliminação de um grande número de poluentes orgânicos perigosos. Segundo Nogueira et al. [15], processos oxidativos avançados (POAs) com utilização de reagentes Fenton apresentam grande eficiência na oxidação de uma ampla variedade de compostos orgânicos.

\subsection{Resíduos Sólidos Urbanos}

Conforme a NBR 10004 [16], entende-se por resíduos sólidos os resíduos nos estados sólido e semi-sólido que resultam de atividades de origem industrial, doméstica, hospitalar, comercial, agrícola e de serviços de varrição. Ultimamente a questão dos resíduos tem sido amplamente discutida na sociedade dentro de várias áreas do conhecimento. A busca de soluções para a destinação final dos resíduos sólidos urbanos é um grande desafio na área ambiental, principalmente, referindo-se a poluição do solo, água e atmosfera. Inovações tecnológicas permitem desenvolver técnicas mais eficientes de disposição final dos resíduos, bem como, alternativas de tratamento de efluentes líquidos e gasosos com melhor aproveitamento de áreas disponíveis para essa finalidade, reduzindo os impactos ambientais.

Os resíduos sólidos apresentam grande diversidade e complexidade quanto a suas características físicas, químicas e biológicas, sendo determinadas de acordo com sua fonte geradora. Tanto a composição quanto a quantidade de resíduos gerados são influenciados por fatores econômicos, sociais, geográficos, educacionais, culturais, tecnológicos e legais. A forma de manejo, tratamento e destino dos resíduos alteram suas características de tal forma que podem causar riscos à saúde [9].

Segundo D’Almeida [17], a porcentagem média nacional da composição dos resíduos sólidos urbanos é 1,6\% vidro, 24,5\% papel, 2,9\% plástico, 2,3\% metais, $52,5 \%$ materiais orgânicos e 16,2\% outros materiais. DEC [18], afirma que para municípios de pequeno porte (menores que 20.000 habitantes), a composição típica média dos resíduos sólidos apresenta $65 \%$ de resíduos orgânicos (sobras de alimentos, folhas, etc.), $15 \%$ de materiais potencialmente recicláveis (papéis, papelão, vidros, plásticos e metais) e $20 \%$ de rejeitos (terra, pedra, madeira, osso, trapos, borracha, couro, etc.). Esses dados evidenciam grande potencial de reciclagem e compostagem de materiais.

Estudos in loco, realizados pelo Laboratório de Engenharia Sanitária e Ambiental (LESA) da Universidade Federal de Viçosa (UFV), em mais de 300 municípios de pequeno porte do Estado de Minas Gerais, indicam que esses municípios geram em média $66,8 \%$ de resíduos orgânicos, $23,9 \%$ de materiais potencialmente recicláveis e $9,3 \%$ de rejeitos [18]. Esses resultados revelam que o potencial de reciclagem e compostagem dos resíduos são ainda maiores do que os apresentados no parágrafo anterior. 


\subsection{Aterro sanitário de resíduos sólidos urbanos}

O aterro sanitário é uma forma adequada de disposição de resíduos sólidos urbanos. De forma sucinta, conforme a NBR 8419 [19], aterro sanitário de resíduos sólidos urbanos consiste em uma técnica de disposição de resíduos sólidos urbanos no solo, sem causar danos ou riscos à saúde pública e à segurança, minimizando os impactos ambientais. A técnica utiliza princípios de engenharia para dispor os resíduos em menor área possível de forma planejada, cobrindo-os com uma camada de terra após uma jornada de trabalho ou em intervalos menores se for necessário.

A decomposição biológica dos resíduos dispostos no aterro sanitário governa a produção de gás e lixiviado. $\mathrm{O}$ percolado, em especial, arrasta com ele substâncias orgânicas e inorgânicas, produtos da decomposição. Suas características dependem do tipo de material disposto no aterro e da fase em que se encontra. Segundo Rodrigues [12], o processo de decomposição dos resíduos sólidos em um aterro sanitário ocorre em três fases: aeróbia, acetogênica e metanogênica. $\mathrm{Na}$ fase aeróbia os microrganismos utilizam o oxigênio ainda disponível juntamente com os resíduos. Essa fase tem curta duração devido ao rápido consumo do oxigênio. Na segunda fase, acetogênica, os microrganismos predominantes são aeróbios facultativos, ou seja, preferencialmente não utilizam oxigênio, porém são tolerantes a ele. Ainda nessa fase dá-se o processo fermentativo. $\mathrm{Na}$ terceira e última fase, metanogênica, os compostos orgânicos começam a ser consumidos estritamente por organismos anaeróbios.

\subsection{Impacto ambiental do lixiviado}

A composição do lixiviado proveniente dos aterros sanitários varia conforme os tipos de resíduos dispostos no aterro. Segundo PROSAB [20], esse efluente pode conter matéria orgânica solubilizada, nutrientes como nitrogênio e fósforo, ácidos orgânicos voláteis, metais pesados como cádmio, zinco e mercúrio, e organoclorados provenientes do descarte de inseticidas e/ou agrotóxicos, além de microorganismos.

Quando lançado diretamente no ambiente o lixiviado de aterro sanitário pode causar danos, principalmente, aos recursos hídricos. Em corpos hídricos o lixiviado pode reduzir a quantidade de oxigênio dissolvido, alterando as condições do ambiente aquático de aeróbio para anaeróbio. Esse tipo de efluente pode causar também a eutrofização de corpos hídricos pelo aporte de nutrientes como nitrogênio e fósforo. Algumas substâncias presentes no efluente podem ser tóxicas ou bioacumulativas, agredindo alguns grupos de organismos aquáticos e até mesmo o homem. Segundo Nascimento Filho et al. [21], cetonas, alcoóis, compostos nitrogenados e ácidos carboxílicos são substâncias normalmente encontradas em lixiviados de aterros sanitários. O autor também afirma que a presença de outros compostos de largo emprego industrial e com reconhecida ação ambiental, indicam que a degradação desse efluente por ação bacteriológica não é eficiente, contribuindo para um aumento do potencial toxicológico do lixiviado produzido.

\subsection{Processos oxidativos avançados (POAs)}

Para o tratamento de substâncias pouco biodegradáveis são utilizados métodos de coagulação química. Os POAs são de grande interesse no tratamento ou pré-tratamento de efluentes que contenham essas substâncias, convertendo matéria orgânica em $\mathrm{CO}_{2}$ e $\mathrm{H}_{2} \mathrm{O}$ ou no casso do pré-tratamento em substâncias com maior biodegradabilidade (Gogate e Pandit apud [22], p. 623). Segundo Aguiar et al. [22], um dos processos oxidativos mais promissores é o Fenton, que consiste na reação entre $\mathrm{Fe}^{2+}$ e $\mathrm{H}_{2} \mathrm{O}_{2}$, gerando radicais $\mathrm{OH}$, que possuem elevado potencial de oxiredução (equação 1 ).

$$
\mathrm{Fe}^{2+}+\mathrm{H}_{2} \mathrm{O}_{2} \rightarrow \mathrm{Fe}^{3+}+{ }^{-} \mathrm{OH}+{ }^{\circ} \mathrm{OH}
$$

Há mais de um século, a oxidação catalítica de ácido tartárico na presença de sais ferrosos e peróxido de hidrogênio foi relatada por Fenton apud [15], p. 401. Quarenta anos após a primeira observação do que seria a chamada "reação de Fenton" (equação 1) (Wardmann apud [15], p. 400), foi proposto que o radical hidroxila é a espécie oxidante neste sistema, capaz de oxidar várias classes de compostos orgânicos em uma reação espontânea que ocorre no escuro (Haber e Weiss apud [15], p.401).

$\mathrm{Na}$ ausência de um substrato o radical hidroxila formado pode oxidar outro íon $\mathrm{Fe}^{2+}$ (equação 2).

$$
\mathrm{Fe}^{2+}+{ }^{\circ} \mathrm{OH} \rightarrow \mathrm{Fe}^{3+}+{ }^{-} \mathrm{OH}
$$

É importante salientar que o ferro em solução aquosa existe como aquo-complexos. No entanto, para simplificação do texto foram omitidas as águas de hidratação nas reações representadas. Os íons férricos formados podem decompor $\mathrm{H}_{2} \mathrm{O}_{2}$ cataliticamente $\mathrm{a}_{2} \mathrm{O}$ e $\mathrm{O}_{2}$, cujos passos são dependentes do $\mathrm{pH}$ (equações 3-7).

$$
\begin{aligned}
& \mathrm{Fe}^{3+}+\mathrm{H}_{2} \mathrm{O}_{2} \leftrightarrow \mathrm{FeOOH}^{2+}+\mathrm{H}^{+} \\
& \mathrm{FeOOH}^{2+} \rightarrow \mathrm{Fe}^{2+}+\mathrm{HO}_{2} \\
& \mathrm{Fe}^{2+}+\mathrm{HO}_{2}^{\cdot} \rightarrow \mathrm{Fe}^{3+}+\mathrm{HO}_{2}^{-} \\
& \mathrm{Fe}^{3+}+\mathrm{HO}_{2}^{\cdot} \rightarrow \mathrm{Fe}^{2+}+\mathrm{O}_{2}+\mathrm{H}^{+} \\
& \mathrm{H}_{2} \mathrm{O}_{2}+{ }^{\circ} \mathrm{OH} \rightarrow \mathrm{HO}_{2}^{\cdot}+\mathrm{H}_{2} \mathrm{O}
\end{aligned}
$$

Como pode ser visto na equação $7, \mathrm{H}_{2} \mathrm{O}_{2}$ pode também atuar como seqüestrador de radical hidroxila, formando o radical hidroperoxila $\left(\mathrm{HO}_{2}{ }^{\circ}\right)$, que tem menor potencial de redução do que ${ }^{\circ} \mathrm{OH}$, prejudicando, portanto, o processo de degradação. Isto ocorre na presença de excesso de $\mathrm{H}_{2} \mathrm{O}_{2}$, pois neste caso, a concentração de $\mathrm{Fe}^{2+}$ no meio é baixa em relação à de $\mathrm{Fe}^{3+}$, uma vez que a reação entre $\mathrm{Fe}^{3+}$ e $\mathrm{H}_{2} \mathrm{O}_{2}$ (equação 3) é muito mais lenta que a decomposição de $\mathrm{H}_{2} \mathrm{O}_{2}$ na presença de $\mathrm{Fe}^{2+}$. $\mathrm{O}$ efeito prejudicial do excesso de $\mathrm{H}_{2} \mathrm{O}_{2}$ na degradação de compostos orgânicos foi observado na degradação de herbicidas e efluentes, o que demanda uma atenção especial para a utilização da concentração adequada (Paterlini e Nogueira apud [15], p.401; Torrades et al. apud [15], p.401). 
Somente após quase um século da publicação do primeiro trabalho envolvendo os regentes de Fenton, os mesmos começaram a ser aplicados na oxidação de contaminantes orgânicos presentes em água, efluente e no solo. Um dos primeiros trabalhos que descreveram a oxidação de compostos orgânicos visando o tratamento de águas por reação de Fenton foi de Barbeni et al. apud [15], p. 401, onde foi estudada a degradação de clorofenóis. A potencialidade do processo para o tratamento de efluentes foi mais tarde enfatizada por Bigda apud [15], p. 401, devido à simplicidade de sua aplicação, uma vez que a reação ocorre à temperatura e pressão ambientes, não requer nenhum reagente ou equipamento especial e se aplica a uma grande variedade de compostos. Além disso, o ferro é o quarto elemento mais abundante na crosta terrestre.

Desde então, um grande número de trabalhos tem demonstrado a aplicabilidade do sistema $\mathrm{H}_{2} \mathrm{O}_{2}$ e sais ferrosos para a degradação de diferentes compostos orgânicos, dentre esses estudos pode-se citar os realizados por: Arruda e Jardim [23]; De Julio et al. [24]; Villa e Nogueira [25]; Tiburtius et at. [26]. Tradicionalmente o $\mathrm{Fe}^{2+}$ é utilizado para catalisar a decomposição do peróxido de hidrogênio na oxidação de compostos orgânicos por reação Fenton. Porém, estudos realizados por Villa e Nogueira [25] mostram que o $\mathrm{Fe}^{3+}$ também pode ser utilizado na oxidação de compostos orgânicos, contudo essa espécie catalisa o peróxido de hidrogênio com menor velocidade do que o $\mathrm{Fe}^{2+}$, reduzindo a eficiência do método. A reação de Fenton permite algumas variações quanto ao metal utilizado na reação, sendo o cobre uma dessas possibilidades. Na também conhecida como reação de Fenton cuprosa, de modo semelhante ao Ferro, o cobre reage com $\mathrm{H}_{2} \mathrm{O}_{2}$ formando complexos intermediários, que em seguida se decompõe formando radical $\mathrm{OH}$. A reação cuprosa é aproximadamente 3 vezes mais rápida que a ferrosa (Wardman e Candeias apud [22], p. 623).

Deste modo o trabalho tem como objetivo avaliar a eficiência do emprego do processo Fenton, com sais de ferro no tratamento do lixiviado de resíduos sólidos urbanos, proveniente de aterro sanitário.

\section{Parte Experimental}

\subsection{Coleta das amostras}

As amostras de lixiviado foram coletadas no aterro controlado do município de Santa Maria - RS, localizado a $29^{\circ} 39^{\prime} 42.59^{\prime \prime} \mathrm{S}$ e $53^{\circ} 52^{\prime} 21.75^{\prime \prime O}$ (Figura 1). Os resíduos sólidos urbanos gerados no município não são mais depositados nesse local, sendo depositados em um aterro sanitário licenciado pela Fundação Estadual de Proteção Ambiental (FEPAM), localizado em área adjacente a do antigo depósito, que foi desativado no inicio de 2008.

Visando obter o efluente com máxima carga de poluentes, o lixiviado foi coletado antes de passar pelo sistema de tratamento existente no aterro, que é composto por três lagoas de estabilização. Na Figura 1 observa-se o local da coleta das amostras, que coincide com o local de entrada do efluente no sistema de tratamento biológico.
Os frascos utilizados na coleta foram previamente limpos com solução de limpeza sulfocrômica e água destilada. Após a coleta os frascos com efluente foram armazenados em refrigerador à temperatura de $4^{\circ} \mathrm{C}$.

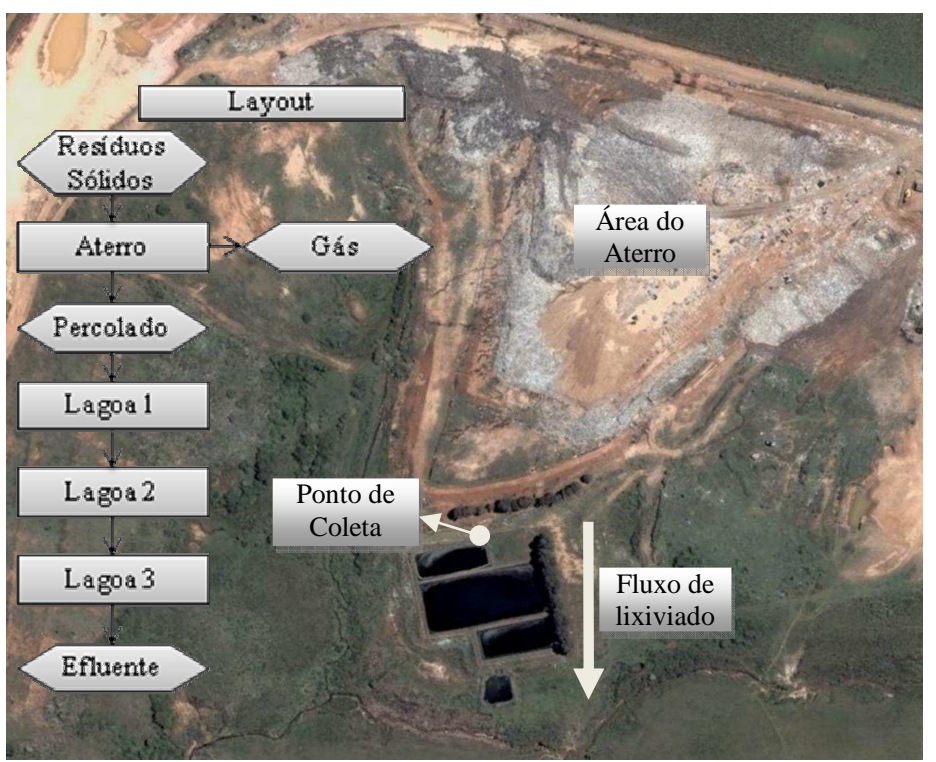

Figura 1 - Imagem e Layout do aterro controlado do município de Santa Maria - RS.

\subsection{Tratamentos}

Foram experimentados cinco tratamentos com diferentes proporções dos reagentes de Fenton, (sulfato de ferro $\left(\mathrm{FeSO}_{4}\right)$ e peróxido de hidrogênio $\left.\left(\mathrm{H}_{2} \mathrm{O}_{2}\right)\right)$ seguindo as relações $\mathrm{H}_{2} \mathrm{O}_{2} / \mathrm{FeSO}_{4}$ de 0,$8 ; 2,0 ; 3,0 ; 4,0$ e 6,0 (Tabela 1).

Com o efluente em um copo de becker, sob agitação, adicionou-se solução $\mathrm{HCl} 15 \%$ (v/v), ajustando-se o pH em aproximadamente 4. Posteriormente foram adicionados o sulfato de ferro $\left(\mathrm{FeSO}_{4}\right)$ e o peróxido de hidrogênio $\left(\mathrm{H}_{2} \mathrm{O}_{2}\right)$ respectivamente, seguindo as proporções indicadas na Tabela 1. Decorridos os 30 minutos de reação, o pH foi neutralizado pela adição de solução de $\mathrm{NaOH} 5 \%$ $(\mathrm{m} / \mathrm{v})$. Com o pH ajustado em aproximadamente 7 , as reações entre $\mathrm{FeSO}_{4}$ e $\mathrm{H}_{2} \mathrm{O}_{2}$ são praticamente cessadas. Por fim o efluente foi filtrado com papel filtro para posterior análise. Todos os experimentos foram mantidos a temperatura ambiente. Na Figura 2 pode-se visualizar o fluxograma do processo realizado. O fator utilizado para investigar a eficiência do processo Fenton no tratamento de lixiviado foi à proporção de $\mathrm{H}_{2} \mathrm{O}_{2}$ em relação ao $\mathrm{FeSO}_{4}$.

Tabela 1 - Volume de efluente, concentração de $\mathrm{H}_{2} \mathrm{O}_{2}$ e $\mathrm{FeSO}_{4}$ e relação $\left(\mathrm{H}_{2} \mathrm{O}_{2} / \mathrm{FeSO}_{4}\right)$ utilizados para as condição de $\mathrm{pH} \approx 4$ e tempo de reação de 30 minutos para cada tratamento

\begin{tabular}{|c|c|c|c|c|}
\hline $\begin{array}{c}\text { Tratamento } \\
\left(\mathrm{TH}_{2} \mathrm{O}_{2} / \mathrm{FeSO}_{4}\right)\end{array}$ & $\begin{array}{c}\text { Efluente } \\
(L)\end{array}$ & $\begin{array}{c}\mathrm{H}_{2} \mathrm{O}_{2} \\
\left(\mathrm{~g} . \mathrm{L}^{-1}\right) \\
\end{array}$ & $\begin{array}{l}\mathrm{FeSO}_{4} \\
\left(\mathrm{~g} . \mathrm{L}^{-1}\right)\end{array}$ & $\mathrm{H}_{2} \mathrm{O}_{2} / \mathrm{FeSO}_{4}$ \\
\hline$T_{0,8}$ & 0,2 & 0,14 & 0,18 & 0,8 \\
\hline$T_{2,0}$ & 0,2 & 0,14 & 0,07 & 2,0 \\
\hline$T_{3,0}$ & 0,2 & 0,55 & 0,18 & 3,0 \\
\hline$T_{4,0}$ & 0,2 & 0,36 & 0,09 & 4,0 \\
\hline$T_{6,0}$ & 0,2 & 1,08 & 0,18 & 6,0 \\
\hline
\end{tabular}




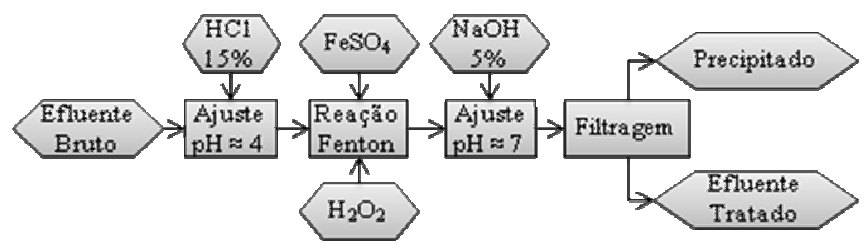

Figura 2 - Fluxograma do experimento de Fenton aplicado em lixiviado de aterro de resíduos sólidos urbanos.

\subsection{Avaliação da eficiência dos tratamentos}

Os parâmetros utilizados na investigação foram: demanda química de oxigênio (DQO), demanda bioquímica de oxigênio $\left(\mathrm{DBO}_{5}\right)$, condutividade elétrica, turbidez e cor.

Esses parâmetros foram determinados para 0 efluente bruto e após a aplicação dos tratamentos $\boldsymbol{T}_{\boldsymbol{0}, \boldsymbol{8}}, \boldsymbol{T}_{\mathbf{2}, \boldsymbol{0}}$, $\boldsymbol{T}_{3,0}, \quad \boldsymbol{T}_{4,0}$ e $\quad \boldsymbol{T}_{6,0} . \quad$ Os parâmetros para avaliação do experimento foram determinados segundo os Standard methods for the examination of water an wastewater da American Public Health Association APHA [27].

A eficiência dos tratamentos foi avaliada tendo como base a porcentagem de remoção dos parâmetros: DQO, $\mathrm{DBO}_{5}$, condutividade elétrica, turbidez e cor. Também foi utilizada a biodegradabilidade do efluente, dada pela relação DQO/DBO. Para calcular a eficiência de remoção, foram quantificados os parâmetros já referidos do efluente bruto e depois de tratado. Para analisar a variância entre os tratamentos foi aplicado o teste estatístico Anova e para avaliar as diferenças entre as médias foi utilizado o teste de Tukey $(\mathrm{P}<0,05)$. Foram coletas 3 amostras em diferentes períodos, para as quais o experimento foi repetido 2 vezes, totalizado 6 repetições do método para cada uma das diferentes proporções de $\mathrm{H}_{2} \mathrm{O}_{2} / \mathrm{FeSO}_{4}$.

\section{Resultados e discussões}

Observam-se nas características do efluente bruto na Tabela 2, altos valores da relação DQO/DBO, indicando que o tratamento físico-químico é adequado para o efluente estudado. Na amostra 3, devido a chuva ocorrida no dia anterior a coleta, houve uma diluição do efluente, alterando conseqüentemente os valores de DQO, DBO, pH, condutividade elétrica e turbidez. Observa-se que ocorreu uma diluição da DQO, DBO e condutividade elétrica, com uma considerável redução da concentração desses parâmetros. Já a turbidez teve um aumento quando comparada as outras duas amostras, fato que deve-se, principalmente, ao carreamento de partículas como silte e argila pela água da chuva. $\mathrm{O}$ pH da amostra 3 aproximou-se do neutro, devido a diluição provocada pela água de chuva, que possui $\mathrm{pH}$ normalmente levemente ácido.
Tabela 2 - Parâmetros determinados nas amostras de lixiviado bruto coletadas no aterro controlado de resíduos sólidos urbanos do município de Santa Maria - RS.

\begin{tabular}{ccccccc}
\hline Amostra & $\begin{array}{c}\mathbf{D Q O} \\
\left(\mathbf{m} \boldsymbol{L}^{-1}\right)\end{array}$ & $\begin{array}{c}\text { DBO } \\
\left(\boldsymbol{m g L}^{-1}\right)\end{array}$ & $\boldsymbol{D Q O} \mathbf{D B O}$ Turbidez Condutividade & $\boldsymbol{p H}$ \\
\hline $\mathbf{1}^{*}$ & 3413,3 & 1085,0 & 3,1 & 84,0 & 14,775 & 8,38 \\
$\mathbf{2}^{*}$ & 4552,0 & 2145,0 & 2,1 & 96,5 & 15,014 & 8,10 \\
$\mathbf{3}^{*}$ & 2800,0 & 880,0 & 3,2 & 117,0 & 6,832 & 7,27 \\
\hline
\end{tabular}

*As amostras foram coletadas em diferentes períodos.

Na Figura 3 podem-se visualizar os valores médios da remoção de DQO dos tratamentos testados. Considerando todos os tratamentos experimentados o processo com reagentes Fenton remove em média $47,9 \%$ da DQO, que é inferior a média de $61,0 \%$ determinada por Lange et al. [11] em condições diferentes das expostas no presente trabalho. $\mathrm{O}$ tratamento que apresentou melhor desempenho na remoção de DQO foi o tratamento $\boldsymbol{T}_{\boldsymbol{0}, 8}$ com remoção média de $54,5 \%$, indicando que ocorreu a mineralização de mais da metade da matéria orgânica presente no efluente. Os tratamentos $\boldsymbol{T}_{\mathbf{3}, \boldsymbol{0}}, \boldsymbol{T}_{\mathbf{4}, \boldsymbol{0}}, \boldsymbol{T}_{\mathbf{2}, \boldsymbol{0}}$ e $\boldsymbol{T}_{\boldsymbol{6}, \boldsymbol{0}}$, removeram em média respectivamente $50,0 \%, 47,6 \%, 45,1 \%$ e $42,5 \%$ da DQO do lixiviado, valores que, segundo o teste estatístico Anova, não diferem significativamente entre si, bem como não diferem significativamente do tratamento $\boldsymbol{T}_{\boldsymbol{0}, \boldsymbol{8}}$.

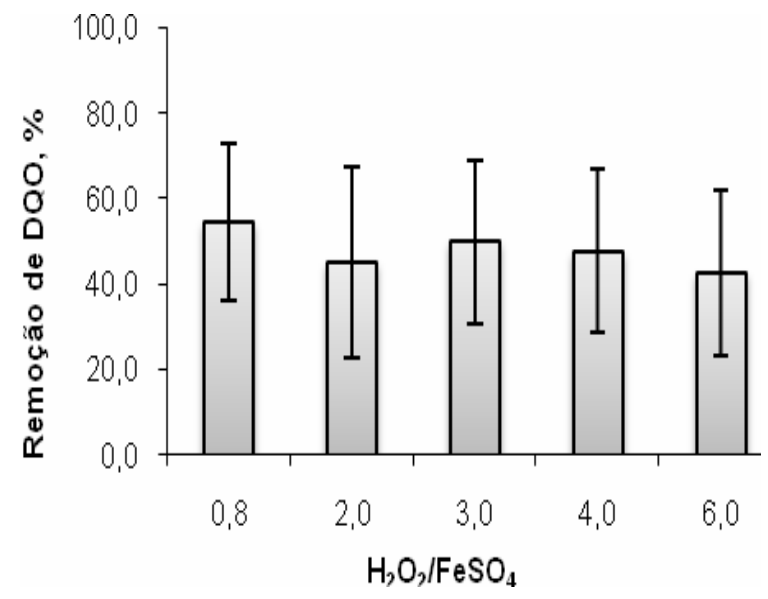

Figura 3 - Porcentagem média e variação da remoção de DQO dos tratamentos aplicados com diferentes proporções dos reagentes $\mathrm{H}_{2} \mathrm{O}_{2}$ e $\mathrm{FeSO}_{4}$.

$\mathrm{Na}$ remoção de $\mathrm{DBO}_{5}$ foram obtidos resultados negativos, ou seja, a demanda bioquímica de oxigênio aumentou após o tratamento. A partir desses resultados pode-se deduzir que ocorreu quebra de parte das substâncias não biodegradáveis em substâncias biodegradáveis, em outras palavras, parte das substâncias que dificilmente seriam degradadas por microorganismos, após o tratamento passam a ser facilmente oxidadas biologicamente. Verificam-se na Figura 4, as médias de remoção de $\mathrm{DBO}_{5} . \mathrm{O}$ tratamento $\boldsymbol{T}_{2,0}$ proporcionou o maior aumento da $\mathrm{DBO}_{5}$, apresentando um acréscimo de 45,9\%. Os demais tratamentos, $\boldsymbol{T}_{\mathbf{4}, \boldsymbol{0}}, \boldsymbol{T}_{\mathbf{3 , \boldsymbol { 0 }},}, \boldsymbol{T}_{\boldsymbol{0}, \boldsymbol{8}}$ e $\boldsymbol{T}_{\boldsymbol{6}, \boldsymbol{0}}$, apresentaram um aumento da $\mathrm{DBO}_{5}$ de $34,5 \%, 33,0 \%, 27,4 \%$ e $15,2 \%$, respectivamente. Contudo não foram evidenciadas diferenças significativas $(\mathrm{P}<0,05)$. 


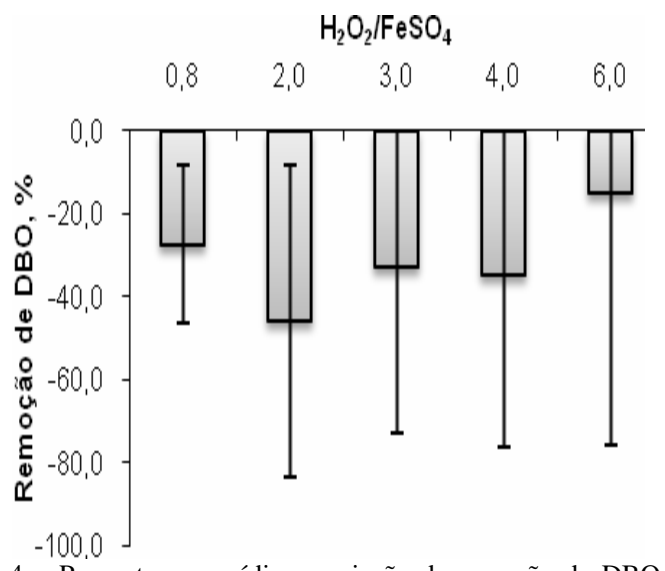

Figura 4 - Porcentagem média e variação da remoção de $\mathrm{DBO}_{5}$ dos tratamentos aplicados com diferentes proporções dos reagentes $\mathrm{H}_{2} \mathrm{O}_{2} \mathrm{e}$ $\mathrm{FeSO}_{4}$.

Na Figura 5 visualizam-se as médias da remoção de turbidez para os tratamentos. Nota-se que apenas o tratamento $\boldsymbol{T}_{4,0}$ não obteve eficiência maior que $50,0 \%$ na remoção de turbidez. Os melhores resultados foram obtidos nos tratamentos $\boldsymbol{T}_{2,0}$ e $\boldsymbol{T}_{3,0}$, que apresentaram $57,8 \%$ e $57,1 \%$ de remoção da turbidez, respectivamente. O tratamento $\boldsymbol{T}_{\boldsymbol{0}, \boldsymbol{8}}$ removeu $55,4 \%$ e o tratamento $\boldsymbol{T}_{6,0} 51,9 \%$. Para a remoção da turbidez não verificaram-se diferenças significativas $(\mathrm{P}<0,05)$ entre os tratamentos

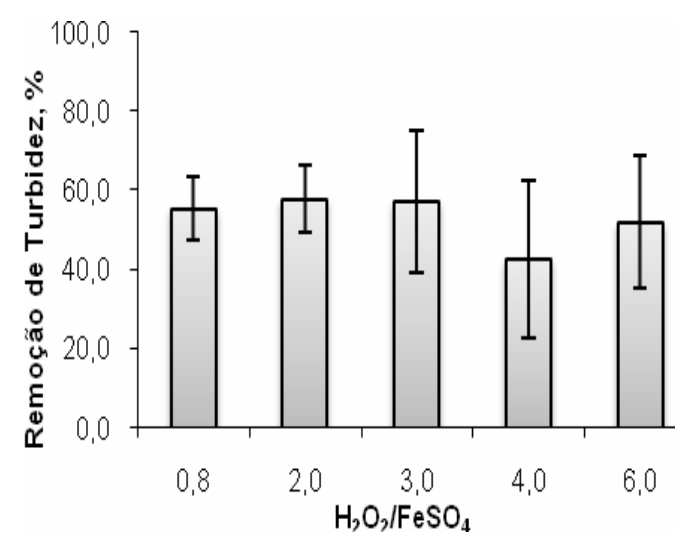

Figura 5 - Porcentagem média e variação da remoção de turbidez dos tratamentos aplicados com diferentes proporções dos reagentes $\mathrm{H}_{2} \mathrm{O}_{2}$ e FeSO,

Os resultados de remoção da condutividade elétrica, seguindo o exemplo da remoção de $\mathrm{DBO}_{5}$, também foram negativos. Em média após a aplicação do processo Fenton a condutividade elétrica do efluente teve um aumento de $27,1 \%$. O aumento da condutividade elétrica pode ser atribuído a mineralização de parte da matéria orgânica presente no efluente, que é o resultado esperado do tratamento. Em outras palavras, a matéria orgânica foi convertida em inorgânica, aumentando a quantidade de íons presentes no efluente, e conseqüentemente aumentando sua condutividade elétrica. As médias de remoção da condutividade elétrica para cada tratamento podem ser visualizadas na Figura 6. Na condutividade elétrica não verificaram-se diferenças significativas $(\mathrm{P}<0,05)$ entre os tratamentos.

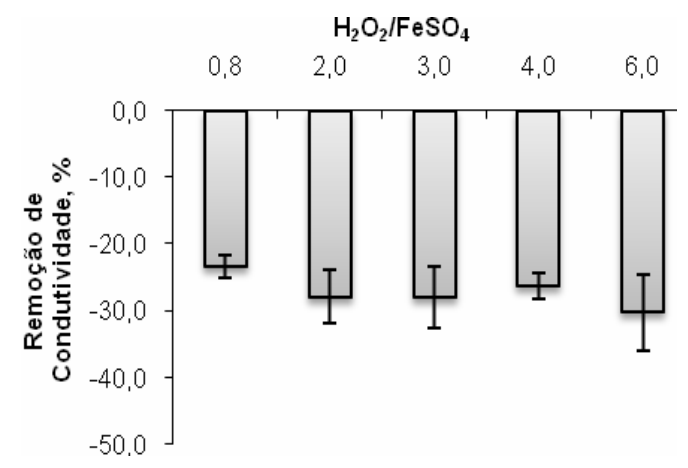

Figura 6 - Porcentagem média e variação da remoção de condutividade elétrica dos tratamentos aplicados com diferentes proporções dos reagentes $\mathrm{H}_{2} \mathrm{O}_{2}$ e $\mathrm{FeSO}_{4}$.

$\mathrm{Na}$ remoção da cor, constatou-se uma redução de até $85,8 \%$, ocorrida no tratamento $\boldsymbol{T}_{6,0}$, que tem a maior concentração de $\mathrm{H}_{2} \mathrm{O}_{2}$ em relação a concentração de $\mathrm{FeSO}_{4}$. O tratamento $\boldsymbol{T}_{3,0}$ apresentou o segundo melhor desempenho na remoção da cor, $74,8 \%$, este seguido dos tratamentos $\boldsymbol{T}_{\boldsymbol{0}, 8}$, $\boldsymbol{T}_{4,0}$ e $\boldsymbol{T}_{2,0}$, que removeram respectivamente $69,9 \%, 69,7 \%$ e 53,0\% (Figura 7). Pelo teste de Tukey verificou-se que na remoção de cor a maior média (tratamento $\boldsymbol{T}_{6,0}$ ) difere significativamente dos tratamentos $\boldsymbol{T}_{\boldsymbol{0}, \boldsymbol{8}}, \boldsymbol{T}_{\mathbf{2}, \boldsymbol{0}}$ e $\boldsymbol{T}_{\mathbf{4}, \boldsymbol{0}}$. A menor média (tratamento $\boldsymbol{T}_{2,0}$ ) difere significativamente dos tratamentos $\boldsymbol{T}_{\boldsymbol{0}, \boldsymbol{8}}, \boldsymbol{T}_{\mathbf{3}, \boldsymbol{0}}, \boldsymbol{T}_{\mathbf{4}, \boldsymbol{0}}$ e $\boldsymbol{T}_{\boldsymbol{6}, \boldsymbol{0}}$. O teste ainda infere que não existe diferença significativa entre os tratamentos $\boldsymbol{T}_{\boldsymbol{0}, \boldsymbol{8}}, \boldsymbol{T}_{\mathbf{3}, \boldsymbol{\theta}} \mathrm{e}$ $\boldsymbol{T}_{4,0}$, bem como, não existe diferença significativa entre os tratamentos $\boldsymbol{T}_{3,0}$ e $\boldsymbol{T}_{6,0}$

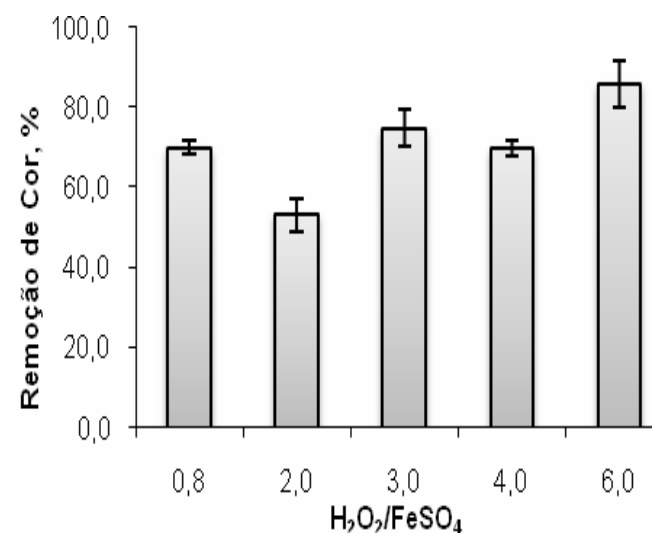

Figura 7 - Porcentagem média e variação da remoção de cor dos tratamentos aplicados com diferentes proporções dos reagentes $\mathrm{H}_{2} \mathrm{O}_{2} \mathrm{e}$ $\mathrm{FeSO}_{4}$.

Na Tabela 3 podem ser observados os valores da biodegradabilidade (DQO/DBO) determinados para o efluente bruto e após a aplicação dos tratamentos. Constatase que a relação DQO/DBO média do efluente bruto é 2,90, que segundo Jardim e Canela [28], caracteriza um efluente pouco biodegradável. Segundo os autores, efluentes com relação DQO/DBO menores que 2,5 são facilmente degradados biologicamente, valores entre 2,5 e 5,0 são pouco biodegradáveis e para valores acima de 5 os processos biológicos têm poucas chances de sucesso. Nota-se ainda na Tabela 3 que o efluente após a aplicação dos tratamentos tem uma menor relação DQO/DBO, ou seja, caracteriza-se como um efluente de maior biodegradabilidade. 
O tratamento que proporcionou a maior biodegradabilidade ao efluente foi o tratamento $\boldsymbol{T}_{\boldsymbol{0}, \boldsymbol{8}}$, reduzindo a relação DQO/DBO de 2,90 para 1,20 , esse tratamento é o que possui a menor relação $\mathrm{H}_{2} \mathrm{O}_{2} / \mathrm{FeSO}_{4}, 0,8$, com concentração de reagentes de $0,14 \mathrm{~g} . \mathrm{L}^{-1}$ de $\mathrm{H}_{2} \mathrm{O}_{2}$ e 0,18 g. $\mathrm{L}^{-1}$ de $\mathrm{FeSO}_{4}$. Os tratamentos $\boldsymbol{T}_{4, \boldsymbol{0}}, \boldsymbol{T}_{2, \boldsymbol{0}}$ e $\boldsymbol{T}_{\mathbf{3}, \boldsymbol{0}}$ reduziram a relação DQO/DBO para 1,28, 1,31 e 1,32 respectivamente, já o tratamento $\boldsymbol{T}_{\boldsymbol{6}, \boldsymbol{0}}$ reduziu para apenas 1,70 . Verificou-se que não existe diferença significativa $(\mathrm{P}<0,05)$ entre as médias da biodegradabilidade do efluente após a aplicação dos tratamentos experimentados.

Tabela 3 - Média e desvio padrão da relação DQO/DBO (biodegradabilidade) do efluente bruto e após a aplicação dos tratamentos

\begin{tabular}{|c|c|c|c|c|c|}
\hline \multirow{2}{*}{$\begin{array}{c}\text { Biodegradabilidad } \\
\text { e } \\
\end{array}$} & \multicolumn{4}{|c|}{ Tratamento $\left(\mathrm{TH}_{2} \mathrm{O}_{2} / \mathrm{FeSO}_{4}\right)$} & \multirow{2}{*}{ Efluente Bruto } \\
\hline & $\mathbf{T}_{0,8}$ & $\mathbf{T}_{2,0}$ & $\begin{array}{ll}\mathbf{T}_{3,0} & \mathbf{T}_{4,0} \\
\end{array}$ & $\mathbf{T}_{6,0}$ & \\
\hline \multirow{2}{*}{ DQO/DBO } & Média & $\begin{array}{cc}1,20 \\
1,3\end{array}$ & $1,32 \quad 1,28$ & 1,70 & 2,90 \\
\hline & $\begin{array}{l}\text { Desvio } \\
\text { Padrão }\end{array}$ & $0,26 \begin{array}{c}0,3 \\
4\end{array}$ & $0,28 \quad 0,25$ & 0,74 & 0,52 \\
\hline
\end{tabular}

Na Figura 8 pode-se visualizar a distribuição dos valores da biodegradabilidade do efluente bruto e após os tratamentos, segundo a definição de Jardim e Canela [28]. Observa-se na figura que das três amostras de lixiviado bruto, duas encontram-se na área de pouca biodegradabilidade e uma na área considerada biodegradável. Após a aplicação dos tratamentos, a relação DQO/DBO foi reduzida a um valor inferior a 2,5, ou seja, o efluente tornou-se mais biodegradável, sendo que apenas uma das repetições do tratamento $\boldsymbol{T}_{\boldsymbol{6}, \boldsymbol{0}}$ permaneceu com a relação DQO/DBO acima de 2,5.

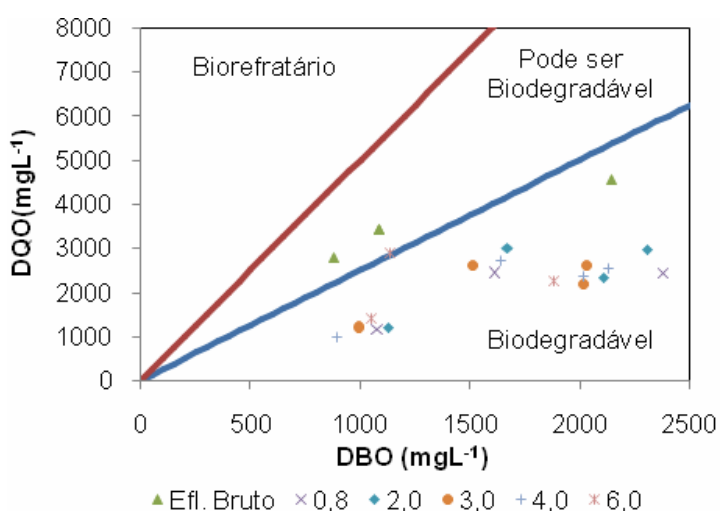

Figura 8 - Distribuição da relação DQO/DBO média do efluente bruto e após os tratamentos $\boldsymbol{T}_{0,8}, \boldsymbol{T}_{2,0}, \boldsymbol{T}_{3,0}, \boldsymbol{T}_{4,0}$ e $\boldsymbol{T}_{6,0}$ na curva de biodegradabilidade segundo a definição de Jardim e Canela [28].

Em valores médios observa-se que a relação DQO/DBO para o lixiviado bruto, está situada na região de baixa biodegradabilidade. Já os valores médios da relação DQO/DBO após o processo Fenton, para todos os tratamentos testados, localizam-se no intervalo que caracteriza efluentes biodegradáveis pela definição de [28]. O tratamento $\boldsymbol{T}_{\boldsymbol{0}, \boldsymbol{8}}$ não foi o que apresentou maior aumento da $\mathrm{DBO}_{5}$ ficando atrás dos tratamentos $\boldsymbol{T}_{\mathbf{2}, \boldsymbol{0}}, \boldsymbol{T}_{\mathbf{4}, \boldsymbol{0}}$ e $\boldsymbol{T}_{3, \boldsymbol{0}}$ respectivamente. Porém o tratamento $\boldsymbol{T}_{0,8}$ destaca-se pela redução da DQO e pelo aumento da biodegradabilidade, ou seja, pelo decremento da relação DQO/DBO do lixiviado, além disso, igualmente ao tratamento $\boldsymbol{T}_{2,0}$ utiliza menor concentração de peróxido de hidrogênio $0,14 \mathrm{~g} \cdot \mathrm{L}^{-1}$.

\section{CONCLUSÕES}

A aplicação do processo Fenton no tratamento de lixiviado de aterros de resíduos sólidos urbanos reduz a demanda química de oxigênio, a turbidez e a cor do efluente nas proporções $\mathrm{H}_{2} \mathrm{O}_{2} / \mathrm{FeSO}_{4}$ de 0,8 a 6,0 .

O tratamento do efluente pela aplicação do processo Fenton aumenta a demanda bioquímica de oxigênio e a condutividade elétrica nas proporções $\mathrm{H}_{2} \mathrm{O}_{2} / \mathrm{FeSO}_{4}$ de 0,8 a 6,0 .

A maior redução na demanda química de oxigênio ocorreu no tratamento com relação $\mathrm{H}_{2} \mathrm{O}_{2} / \mathrm{FeSO}_{4}$ igual a 0,8 , apresentando, também, maior aumento da biodegradabilidade.

O processo oxidativo avançado com reagentes Fenton pode ser utilizado no pré-tratamento de lixiviado de aterros sanitários de resíduos sólidos urbanos.

O processo Fenton aplica-se a uma grande variedade de efluentes, não exigindo complexidade operacional e de equipamentos em seu emprego, além disso, ocorre em condições de temperatura e pressão ambientes. A sua utilização como pré-tratamento de um sistema biológico pode reduzir os riscos de insucesso no tratamento do lixiviado.

\section{Agradecimentos}

Os autores agradecem ao Laboratório de Engenharia Ambiental (LABEA) do Centro Universitário Franciscano (UNIFRA), Santa Maria - RS pelo auxílio técnico e pela cessão de equipamentos e reagentes.

\section{URBAN SOLID WASTE LEACHATE TREATED BY FENTON PROCESS}

ABSTRACT: The leachate generated on sanitary landfill by solid waste decomposition with rainfall water infiltration has high potential of pollution, due to high concentration of toxic substances and low biodegradability. The chemistry coagulation can be an alternative to treat effluents with these characteristics. The work aimed to evaluate the efficiency of Fenton process in the treatment of leachate landfill from urban solid waste. The leachate samples were collected from the controlled landfill of Santa Maria Council - RS. In the experiment were tested five treatments with different proportions of reagents $\left(\mathrm{H}_{2} \mathrm{O}_{2}\right.$ e $\left.\mathrm{FeSO}_{4}\right) \boldsymbol{T}_{\text {H2O2/FeSO4: }} \boldsymbol{T}_{\boldsymbol{0}, \boldsymbol{8}}$, $\boldsymbol{T}_{2,0}, \boldsymbol{T}_{3,0}, \boldsymbol{T}_{4,0}$ and $\boldsymbol{T}_{6,0}$. The experimental data with Fenton process showed an average removing efficiency on chemical oxygen demand (COD) of $47.9 \%$, on the turbidity $53.0 \%$ and $70.7 \%$ for color. The average values of biochemical oxygen demand (BOD) and electric conductivity increased on $31.3 \%$ and $27.1 \%$, respectively. On the leachate treatment with $\boldsymbol{T}_{\boldsymbol{0}, 8}$ notes the largest removal COD and higher increase 
biodegradability. The Fenton process applied to leachate treatment reduces the COD and increases the BOD, in the other words, increase the effluent biodegradability. This process can be used as a pre-treatment on a biological system for sanitary landfill effluents.

Keywords: leachate. effluent. biodegradability. advanced oxidative process. sanitary landfill.

\section{REFERÊNCIAS}

[1] GUERRA, A. J. T.; MARÇAL, M. dos S.; Geomorfologia ambiental; Bertrand Brasil; Rio de Janeiro, 2006. 192p.

[2] IBGE - Instituto Brasileiro de Geografia e Estatística; Pesquisa nacional de saneamento básico 2000; IBGE; Rio de Janeiro, 2002. 397p.

[3] IBAM - Instituto Brasileiro de Administração Municipal; Gestão Integrada de Resíduos Sólidos: manual de gerenciamento integrado de resíduos sólidos; IBAM; Rio de Janeiro, 2001. 200p.

[4] BRASIL. Economia cresce $6 \%$ no primeiro semestre. Secretaria de Comunicação Social da Presidência da República: em questão, Brasília/DF 10 set. 2008. N.699. Notícias. Disponível em: http://www.brasil.gov.br/noticias/em_questao/.questao/eq699/view?searchte rm=consumo. Acesso em 02/10/2008.

[5] VENINO, A. R.; Ministério das Cidades e IBGE realizam Pesquisa Nacional de Saneamento Básico 2008. Assessoria de Comunicação Ministério das Cidades, Brasília/DF, 24 set. 2008. Notícias. Disponível em: http://www.cidades.gov.br/noticias/ mcidades-e-ibge-realizam-pesquisanacional-de-saneamento-basico-2008/. Acesso em 01/10/2008.

[6] PROSAB - Programa de Pesquisa em Saneamento Básico; Alternativas de disposição de resíduos sólidos urbanos para pequenas comunidades; Rima artes e textos; Florianópolis, 2003. 294p.

[7] BAIRD, C.; Química Ambiental; University of Western Ontario. Trad. Maria Angeles Lobo Rocio e Luiz Carlos Marques Carea; 2.ed.; Bookman; Porto Alegre, 2002. 624p.

[8] TARTARI, Leori. Carlos. Avaliação do processo de tratamento do chorume do aterro sanitário de novo Hamburgo. 2003. Dissertação (Programa de Pós-graduação em Engenharia - Energia, Ambiente e Materiais - Mestrado) - Universidade Luterana do Brasil, Novo Hamburgo, 2003. 11f. Disponível em: http://www.li berato.com.br/upload/arquivos/ 0131010716431716.pdf. Acesso em 29/07/2008.

[9] PROSAB - Programa de Pesquisa em Saneamento Básico; Tratamento, recuperação e disposição de resíduos urbanos, com ênfase na proteção dos corpos d'água; Sermografi artes gráficas e editora Ltda; Florianópolis, 2006. 494p

[10] SISINNO, C. L. S.; OLIVEIRA, R. M. de; Resíduos sólidos, ambiente e saúde: uma visão multidisciplinar. Rio de Janeiro: Editora Fiocruz, 2000. $138 \mathrm{p}$.

[11] LANGE, L. C.; ALVES, J. F.; AMARAL, M. C. S.; JÚNIOR, W. de R. M.; Engenharia Sanitária Ambiental, vol. 11, p. 175-183, 2006.

[12] RODRIGUES, Flávia Simões Ferreira. Aplicação da ozonização e do reativo de Fenton como pré-tratamento de chorume com os objetivos de redução da toxicidade e do impacto no processo biológico. 2004, 79f. Dissertação (Programa de Engenharia Civil, área de Recursos Hídricos) Universidade Federal do Rio de Janeiro, Rio de Janeiro. 2004.

[13] MORAIS, J. L.; SIRTORI, C.; PERALTA-ZAMORA, P. G.; Química Nova, v. 29, p. 20-23, 2006.

[14] SANTOS, L. P.; COELHO, M. G. Estudo da eficiência do reagente de Fenton no tratamento de líquidos percolados provenientes do aterro sanitário do município de Uberlândia - MG/Brasil. In: $22^{\circ}$ CONGRESSO BRASILEIRO DE ENGENHARIA SANITÁRIA E AMBIENTAL; V FEIRA INTERNACIONAL DE TECNOLOGIA DE SANEAMENTO AMBIENTAL, 2003, Joinville. Saneamento Ambiental: ética e responsabilidade social. Joinville: ABES, 2003, p. 1-13. Disponível em: http://www.bvsde.paho.org/bvsacd/abes22/cdxx.pdf. Acesso em 10/11/2008.

[15] NOGUEIRA, R. F. P.; TROVÓ, A. G.; SILVA, M. R. A. da; VILLA, R. D.; OLIVEIRA, M. C. de. Química Nova, v. 30, p. 400-408, 2007.

[16] ASSOCIAÇÃO BRASILEIRA DE NORMAS TÉCNICAS - ABNT. Resíduos sólidos - classificação. NBR - 10004. São Paulo: ABNT, 2004.

[17] D’ALMEIDA, M. L. O.; Lixo Municipal: Manual de Gerenciamento Integrado. 2 ed.; IPT/CEMPRE; São Paulo, 2000. 246p.

[18] DEC - Departamento de Engenharia Civil; Identificação e sistematização de tecnologias de baixo custo para o tratamento dos resíduos sólidos urbanos; Universidade Federal de Viçosa; Viçosa-MG, 2002. Disponível em: http://www.cepis.ops-oms.org/bvsars/ p/fulltext/identi/ identi.html. Acesso em 22/04/2008.

[19] ASSOCIAÇÃO BRASILEIRA DE NORMAS TÉCNICAS - ABNT. Apresentação de projetos de aterros sanitários de resíduos sólidos urbanos NBR - 8419, São Paulo: ABNT, 1984.

[20] PROSAB - Programa de Pesquisa em Saneamento Básico; Gerenciamento de resíduos sólidos com ênfase na proteção de corpos d'água: prevenção, geração e tratamento de lixiviado de aterro sanitário; Sermografi artes gráficas e editora Ltda; Florianópolis, 2006. 494p.

[21] NASCIMENTO FILHO, I; MÜHLEN, C. von; CARAMÃO, E. B.; Química Nova, vol. 24, p. 554-556, 2001.

[22] AGUIAR, A.; FERRAZ, A.; CONTRERAS, D.; RODRÍGUEZ, J.; Química Nova, vol. 30, p. 623-628, 2007.

[23] ARRUDA, T. L. de; JARDIM, W. F.; Química Nova, vol. 30, p. 16281632, 2007.

[24] DE JULIO, M.; NEVES E. F. de A.; TROFINO J. C.; BERNARDO L. D.; Engenharia Sanitária Ambiental, vol. 11, p. 260-268, 2006.

[25] VILLA, R. D.; NOGUEIRA, R. F. P.; Eclética Química, vol. 30, p. 69$76,2005$.

[26] TIBURTIUS, E. R. L.; PERALTA-ZAMORA, P.; EMMEL, A.; LEAL, E. S.; Química Nova, vol. 28, p. 61-64, 2005.

[27] APHA - American Public Health Association. Standard methods for the examination of water an wastewater. Waschington, 1999.

[28] JARDIM, W. F. CANELA, M. C.; Caderno Temático: Fundamentos da Oxidação Química no Tratamento de efluentes e remediação de solos; v.1; UNICAMP; Campinas, 2004. 